\title{
FONA-7, a Novel Extended-Spectrum $\beta$-Lactamase Variant of the FONA Family Identified in Serratia fonticola
}

\author{
Danny Fuentes-Castillo, ${ }^{1}$ Pablo Power, ${ }^{2}$ Louise Cerdeira, ${ }^{3}$ Adriana Cardenas-Arias, ${ }^{4}$ Quézia Moura, ${ }^{4}$ \\ Flavio A. Oliveira, ${ }^{5}$ Carlos E. Levy, ${ }^{5}$ Gabriel Gutkind, ${ }^{2}$ José L. Catão-Dias, ${ }^{1}$ and Nilton Lincopan ${ }^{3,4}$
}

\begin{abstract}
Serratia fonticola is a human pathogen widely found in the environment, with birds being reported as possible natural hosts. During an epidemiological and genomic surveillance study conducted to monitor the occurrence of extended-spectrum $\beta$-lactamase (ESBL)-producing Enterobacterales in South American wild birds, we identified an ESBL-positive $S$. fonticola in a fecal sample collected from a Hudsonian Whimbrel, during its nonbreeding range on the Pacific Coast of Chile. Whole genome sequencing analysis and "in silico" modeling revealed a novel variant of the class A ESBLs FONA family, designated FONA-7, which shows 96.28\% amino acid identity with FONA-6; with amino acid substitutions occurring in the signal peptide sequence (Thr22 $\rightarrow$ Ser), and in the mature protein (Ser39 $\rightarrow$ Asn and Thr227 $\rightarrow$ Ile). This finding denotes that migratory birds can be potential vectors for the transboundary spread of ESBL-producing bacteria, creating a further theoretical risk for the origin of novel plasmid-encoded $\beta$-lactamases.
\end{abstract}

Keywords: ESBL, $b l a_{\mathrm{FONA}-7}$, wild bird, South America

\section{Introduction}

$S$ ERRATIA fonticola IS A MEMBER OF THE Yersiniaceae family, order Enterobacterales; widely found in the environment (i.e., drinking water, sewage, and soil), with birds being reported as possible natural hosts. ${ }^{1,2}$ As human pathogen, S. fonticola has been associated with diarrhea, septic arthritis, and wound, respiratory, urinary tract, bloodstream, or skin and soft tissue infections. ${ }^{1,2}$ Resistance to $\beta$-lactams in $S$. fonticola has been mediated by chromosomal class A extended-spectrum $\beta$-lactamases (ESBLs) belonging to the FONA family., ${ }^{3,4}$ To date, six variants of the FONA gene ( $b l a_{\text {FONA-1 }}$ to $b l a_{\text {FONA-6 }}$ ) have been reported (GenBank accession numbers: AJ251239.1-AJ251244.1). We hereby report FONA-7, a novel FONA-type ESBL identified in a $S$. fonticola strain isolated from the migratory shorebird Hudsonian Whimbrel (Numenius phaeopus hudsonicus), in South America.

\section{Materials and Methods}

Identification of the isolate and antimicrobial susceptibility testing

During an epidemiological and genomic surveillance study conducted to monitor the occurrence of ESBL- producing Enterobacterales in South American wild birds $(n=58)$ (Supplementary Table S1), we isolated a Gramnegative and lactose-positive bacteria that grows on MacConkey agar supplemented with ceftriaxone $(2 \mu \mathrm{g} / \mathrm{mL})$, from a fecal sample of a wild bird (Hudsonian Whimbrel), during its non-breeding range on the Pacific Coast of Chile $\left(35^{\circ} 30^{\prime} \mathrm{S}, 72^{\circ} 31^{\prime} \mathrm{W}\right)$. Initially, bacterial identification and susceptibility profile were determined by Vitek 2 system (bioMérieux, Inc., Hazelwood, MO), with further species confirmation by matrix-assisted laser desorption ionization time-of-flight mass spectrometry system (Bruker Daltonik) and susceptibility profile determined by disk diffusion, and $E$ test methods. ${ }^{5,6}$ ESBL production was confirmed by double disk synergy test, whereas additional production of AmpC $\beta$-lactamase was detected by disk potentiation method using 3-amino phenyl boronic acid (APB) with cefoxitin and cefoxitin-APB disks.

Whole genome sequencing analysis, alignment of FONA-type protein sequences, and in silico modeling

The total genomic DNA of $S$. fonticola PE1 was extracted and used to construct a paired-end library, which was

\footnotetext{
${ }^{1}$ Department of Pathology, School of Veterinary Medicine and Animal Sciences, University of São Paulo, São Paulo, Brazil.

${ }^{2}$ Instituto de Investigaciones en Bacteriología y Virología Molecular (IBaViM), Facultad de Farmacia y Bioquímica, Universidad de Buenos Aires, Buenos Aires, Argentina.

${ }^{3}$ Department of Clinical Analysis, Faculty of Pharmacy, University of São Paulo, São Paulo, Brazil.

${ }^{4}$ Department of Microbiology, Institute of Biomedical Sciences, University of São Paulo, São Paulo, Brazil.

${ }^{5}$ Hospital de Clínicas, Universidade Estadual de Campinas, Campinas, Brazil.
} 
sequenced using the MiSeq platform (Illumina) with $2 \times 300 \mathrm{bp}$ sequence length. De novo genome assembly was carried out using SPAdes v3.13.1, ${ }^{8}$ and automatic annotation was performed using Prokka v1.13.3 (www.github.com/tseemann/ prokka). Antibiotic resistance genes and plasmid replicons were identified using ResFinder v3.2 and PlasmidFinder v2.1, respectively. ${ }^{9,10}$ The alignment of all known FONA-type predicted protein sequences (i.e., FONA-1 [CAB61635.1], FONA-2 [CAB61637.1], FONA-3 [CAB61639.1], FONA4 [CAB61641.1], FONA-5 [CAB61643.1], and FONA-6 [CAB61645.1]) was performed using Clustal Omega v1.2.4 (https://www.ebi.ac.uk/Tools/msa/clustalo), whereas in silico modeling of the FONA ESBL in combination with cefotaxime and ceftazidime was performed using Swiss-Model, ${ }^{11}$ Yasara, ${ }^{12}$ and PyMol. ${ }^{13}$

\section{Results}

Identification of the isolate and antibiotic susceptibility profile

Bacterial cultures yielded growth of a $S$. fonticola designated PE1 strain, which displayed resistance to ampicillin, amoxicillin, ticarcillin, piperacillin, amoxicillin-clavulanic acid, ampicillin-sulbactam, ticarcillin-clavulanic acid, aztreonam, cefoxitin, cephalexin, cephalothin, cephazolin, cefaclor, cefuroxime, cefixime, cefoperazone, cefotaxime, ceftazidime, ceftriaxone, ceftiofur, cefepime, and cefpodoxime (Table 1). In addition, $S$. fonticola PE1 exhibited an intermediate susceptibility to piperacillin/tazobactam, remaining susceptible to ertapenem, imipenem, meropenem, doripenem, gentamicin (minimal inhibitory concentration $[\mathrm{MIC}]=0.25 \mu \mathrm{g} / \mathrm{mL})$, amikacin $(\mathrm{MIC}=1.5 \mu \mathrm{g} / \mathrm{mL})$, streptomycin ( $\mathrm{MIC}=6 \mu \mathrm{g} / \mathrm{mL})$, nalidixic acid (MIC $\leq 2 \mu \mathrm{g} / \mathrm{mL}$ ), ciprofloxacin (MIC $\leq 0.25 \mu \mathrm{g} / \mathrm{mL}$ ), enrofloxacin ( $\mathrm{MIC}=$ $0.19 \mu \mathrm{g} / \mathrm{mL}$ ), norfloxacin (MIC $\leq 0.5 \mu \mathrm{g} / \mathrm{mL}$ ), chloramphenicol $(\mathrm{MIC}=2 \mu \mathrm{g} / \mathrm{mL})$, tetracycline $(\mathrm{MIC}=2 \mu \mathrm{g} / \mathrm{mL})$, and trimethoprim/sulfamethoxazole (MIC $\leq 2 / 38 \mu \mathrm{g} / \mathrm{mL}$ ).

\section{Genomic background of ESBL-producing S. fonticola $P E 1$ strain and identification of the novel FONA-type ESBL}

Whole genome sequencing analysis of $S$. fonticola PE1 revealed the presence of a novel FONA-type $\beta$-lactamase, named FONA-7, as assigned by National Center for Biotechnology Information (GenBank accession number: MN634199), with a $96.28 \%$ amino acidic identity to FONA6 (GenBank accession number: NG_049097.1), and no plasmids were detected. In addition, S. fonticola PE1 carried a chromosomal AmpC, which shared 99\% identity with intrinsic class C $\beta$-lactamases identified in $S$. fonticola (GenBank accession number WP_065685009.1). The alignment of all FONA-type predicted protein sequences revealed three amino acid substitutions in FONA-7, in comparison with FONA-6, with one occurring in the signal peptide sequence $(\mathrm{Thr} 22 \rightarrow \mathrm{Ser})$ and two in the mature protein (Ser39 $\rightarrow$ Asn and Thr227 $\rightarrow$ Ile) (Fig. 1). Both substitutions occurring in the mature chain are in the solvent-accessible surface of the protein (Fig. 2), for which they are supposed to not contribute to modifications in the $\beta$-lactamase activity. In silico modeling of FONA-7 in combination with cefotaxime and ceftazidime showed that
Table 1. Susceptibility Profile and Minimal INHIBITORY CONCENTRATIONS OF $\beta$-LACTAM Antibiotics for SeRRatia Fonticola Strain PE1 Carrying the Novel Extended-Spectrum $\beta$-LACTAMASE FONA-7 VARIANT

\begin{tabular}{ll}
\hline -Lactam antibiotics & $\begin{array}{c}\text { Interpretative category } \\
(\text { MIC }, \mu g / m L)^{\mathrm{a}}\end{array}$ \\
\hline Ampicillin & $\mathrm{R}(>256)^{\mathrm{d}}$ \\
Amoxicillin & $\mathrm{R}(>32)^{\mathrm{c}}$ \\
Ticarcillin & $\mathrm{R}^{\mathrm{b}}$ \\
Piperacillin & $\mathrm{R}^{\mathrm{b}}$ \\
Amoxicillin/clavulanic acid & $\mathrm{R}(\geq 32 / 16)^{\mathrm{c}}$ \\
Ampicillin/sulbactam & $\mathrm{R}^{\mathrm{b}}$ \\
Ticarcillin/clavulanic acid & $\mathrm{R}^{\mathrm{b}}$ \\
Piperacillin/tazobactam & $\mathrm{I}(32 / 4)^{\mathrm{c}}$ \\
Aztreonam & $\mathrm{R}(256)^{\mathrm{d}}$ \\
Cefoxitin & $\mathrm{R}(64)^{\mathrm{d}}$ \\
Cephalexin & $\mathrm{R}(>32)^{\mathrm{c}}$ \\
Cephalothin & $\mathrm{R}(256)^{\mathrm{d}}$ \\
Cephazolin & $\mathrm{R}(>32)^{\mathrm{c}}$ \\
Cefaclor & $\mathrm{R}(>32)^{\mathrm{c}}$ \\
Cefuroxime & $\mathrm{R}(64)^{\mathrm{c}}$ \\
Cefixime & $\mathrm{R}(256)^{\mathrm{d}}$ \\
Cefoperazone & $\mathrm{R}(>64)^{\mathrm{c}}$ \\
Cefotaxime & $\mathrm{R}(256)^{\mathrm{d}}$ \\
Ceftazidime & $\mathrm{R}(32)^{\mathrm{d}}$ \\
Ceftriaxone & $\mathrm{R}(256)^{\mathrm{c}}$ \\
Ceftiofur & $\mathrm{R}(>32)^{\mathrm{c}}$ \\
Cefpodoxime & $\mathrm{R}^{\mathrm{b}}$ \\
Cefepime & $\mathrm{R}(256)^{\mathrm{d}}$ \\
Doripenem & $\mathrm{S}^{\mathrm{b}}$ \\
Ertapenem & $\mathrm{S}(\leq 0.5)^{\mathrm{c}}$ \\
Imipenem & $\mathrm{S}(0.75)^{\mathrm{d}}$ \\
Meropenem & $\mathrm{S}(0.064)^{\mathrm{d}}$ \\
\hline
\end{tabular}

${ }^{a}$ Interpretative categories according to CLSI documents. ${ }^{5,6}$

${ }^{\mathrm{b}}$ Susceptibility profile determined by disk diffusion method.

${ }^{\mathrm{c}}$ MICs determined by Vitek 2 system.

${ }^{\mathrm{d}}$ MICs determined by $E$ test method.

CLSI, Clinical and Laboratory Standards Institute; MICs, minimal inhibitory concentrations.

this $\beta$-lactamase is able to efficiently accommodate and hydrolyze oxyimino-cephalosporins (Fig. 2), in accordance with the observed phenotypic behavior.

\section{Discussion}

The antimicrobial resistance (AMR), supported by the vertical and/or horizontal transfer of antibiotic resistance genes, is a serious public health challenge globally. ${ }^{9} \mathrm{Al}-$ though, AMR has been widely associated with pathogens in clinical settings, it is becoming increasingly recognized that nonclinical environments and nonhuman host may also be reservoirs of AMR genes. ${ }^{14}$ In this regard, several studies have confirmed that migratory birds can carry bacterial species harboring clinically significant resistance genes acting, therefore, as reservoir and potential vectors in the global dissemination of antibacterial resistance. ${ }^{14-17}$

In this study, we report a novel ESBL variant of the FONA family, designated FONA-7, in a $S$. fonticola isolated from Hudsonian Whimbrel, a large migratory shorebird that breed in North America and migrates annually to South America, ${ }^{18}$ confirming that migratory birds can be potential vectors for the transboundary spread of ESBL-producing bacteria. 


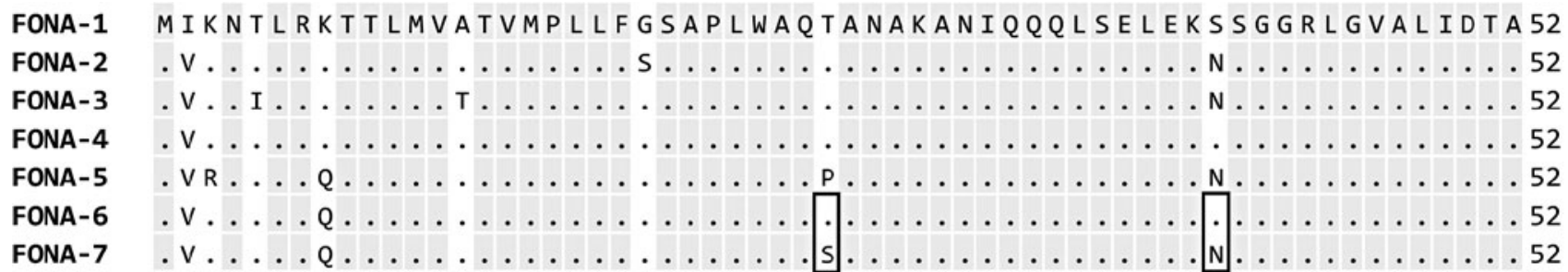

FONA-1 DNSQILYRGDERFPMCISTSKIVMAVSALLKQSETDKNLLAKRMEIKQSDLVNYNPIAEKHL 113

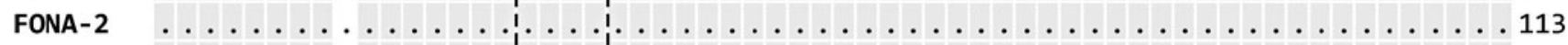

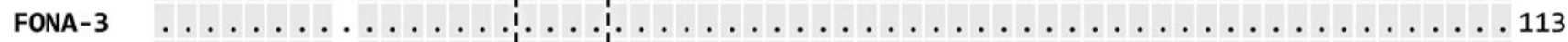

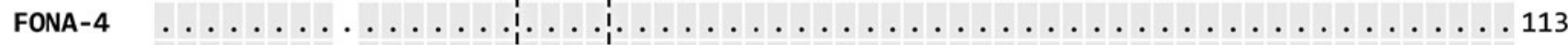

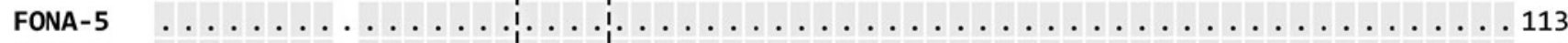

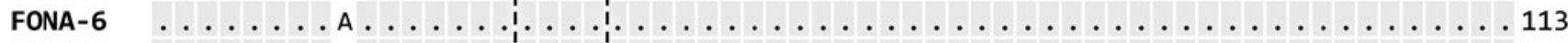

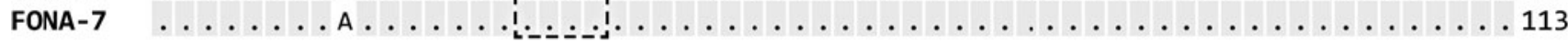

FONA-1 DTGMTLAEFSAAT I QYYSDNTT AMNKILEHLGGPAKVTEFARTIGDKTFRLDRTEPTLNTAI 173

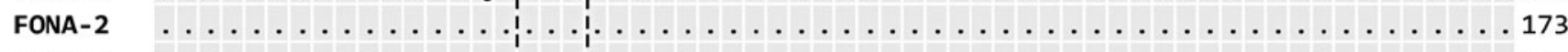

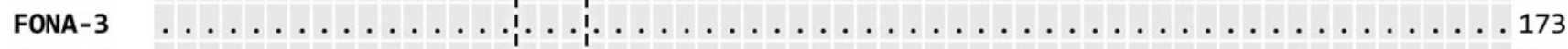

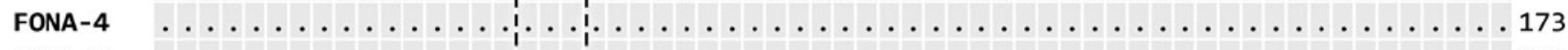

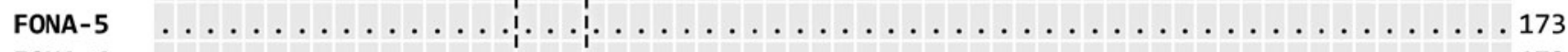

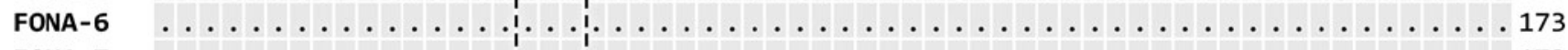

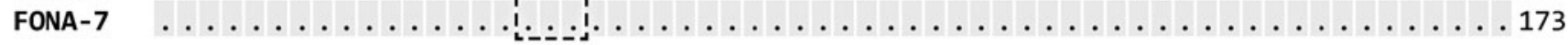

FONA-1 PGDERDTSSPLAMAKSLQNLTLGKALGEPQRAQLVEWMKGNTTGGASIRAGLPT TWIVGD 233

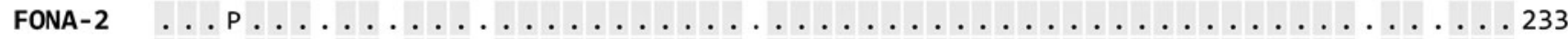

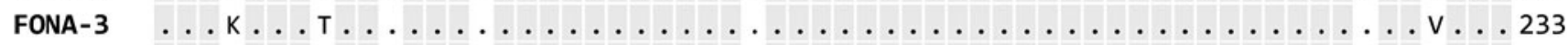

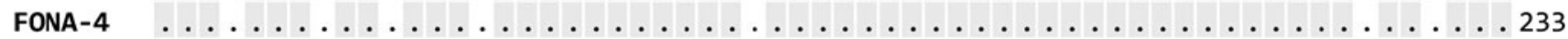

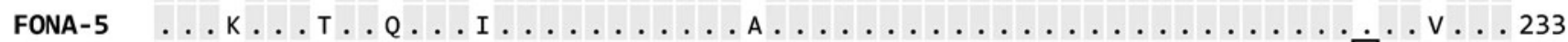

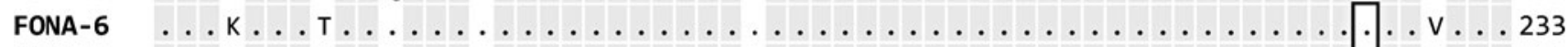

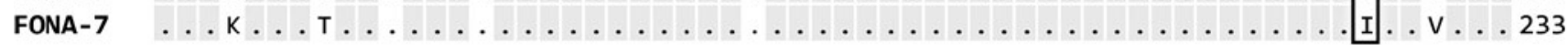

FONA-1 KTGSGDYGTTNDIAVIWPANHAPLVLVTYFTQPQQNAEARKDVLAAAAKIVTEGL 290

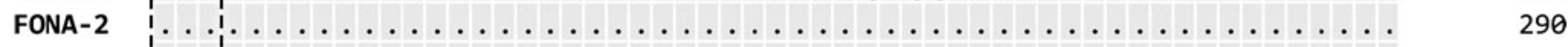

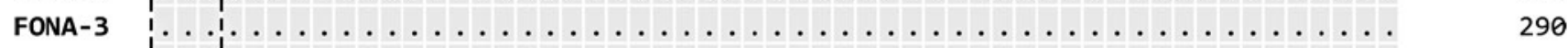

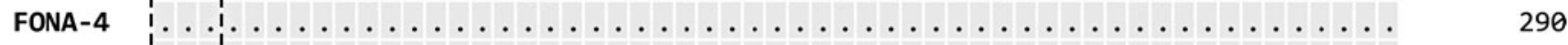

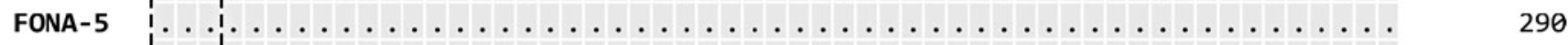

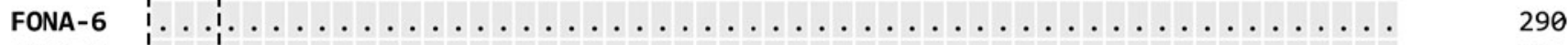

FONA-7

FIG. 1. Multiple sequence alignment of FONA-type ESBLs, including FONA-1 (GenBank accession number: CAB61635.1), FONA-2 (GenBank accession number: CAB61637.1), FONA-3 (GenBank accession number: CAB61639.1), FONA-4 (GenBank accession number: CAB61641.1), FONA-5 (GenBank accession number: CAB61643.1), FONA-6 (GenBank accession number: CAB61645.1), and FONA-7 (this study, GenBank accession number: MN634199). Conserved motifs of class A $\beta$-lactamase are indicated by dashed squares. Amino acid substitutions (Thr22 $\rightarrow$ Ser; Ser39 $\rightarrow$ Asn; and Thr227 $\rightarrow$ Ile) in FONA-7 are indicated by solid squares. ESBLs, extended-spectrum $\beta$-lactamases.

Currently, next-generation sequencing technologies along with sophisticated bioinformatics platforms have improved the diagnostic of bacterial infections and bacterial resistance surveillance. ${ }^{19-21}$ Specifically, the in silico prediction of resistome from bacterial genomes has allowed to track the origin of clinically relevant resistance genes, monitoring their further spread among bacterial population. In this regard, user-friendly and high-quality openly available bioinformatic tools that use database that captures AMR genes from whole-genome data sets are currently available for bacterial genome analyses. Therefore, it is crucial that novel resistance genes, including bla $a_{\mathrm{ESBL}}$ variants, are identified and published, to keep AMR gene repositories as updated as possible. ${ }^{19-21}$

Although, lack of enzyme kinetics data can be considered as limitation of this study, the FONA-7 ESBL activity could be evidenced by the resistance phenotype to penicillins and broad-spectrum cephalosporins (Table 1). However, additional studies evaluating the kinetics of FONA-7 are necessary to better understand the biochemical characteristics 

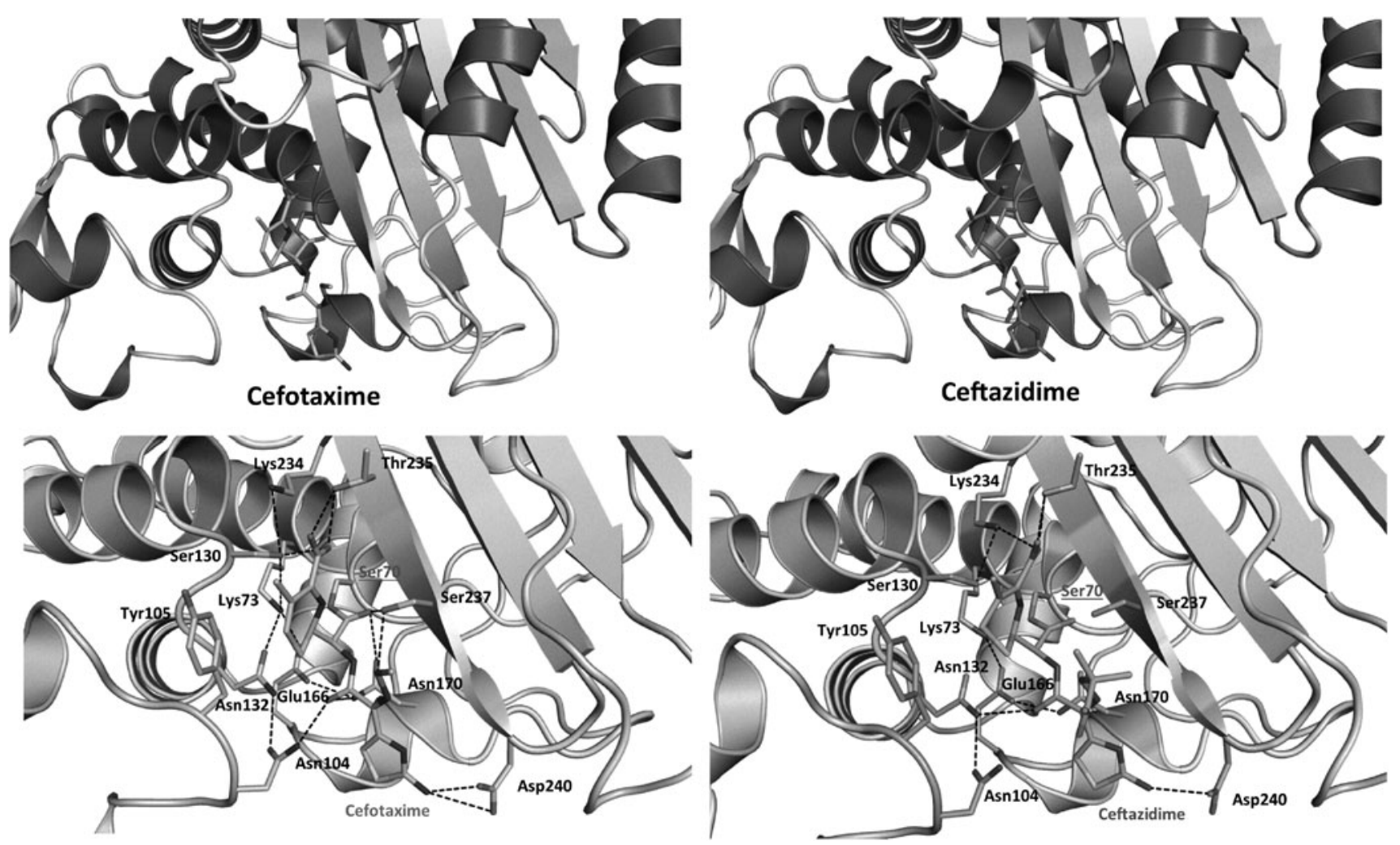

FIG. 2. Modeled structure of FONA-7 extended-spectrum $\beta$-lactamase. Upper panel, in silico models showing the overall structure of FONA-7 $\beta$-lactamase in complex with cefotaxime and ceftazidime. Lower panel, detail of the active site and the main hydrogen bonds (dashed lines) involved in the acyl-enzyme complexes with both cefotaxime and ceftazidime.

of this novel FONA-type ESBL variant. In contrast, although this finding denotes a theoretical risk for the origin of a novel plasmid-encoded enzyme in migratory birds and in its trans-American flyway, more research must be done to determine whether an exchange of chromosomal FONA ESBL gene occurs between $S$. fonticola and other enterobacterial species in the gut of human and other animal hosts.

\section{Acknowledgments}

We thank Daniela Castro, Alex Fuentes, and Camilo Fuentes for the help in collecting samples; Herrison Fontana for the help in data analysis; Dra Silvia Santos for Vitek 2 assistance; Cefar Diagnóstica Ltda. (Brazil) for kindly supplying antibiotic disks for susceptibility testing; and CEFAP-GENIAL facility for Illumina sequencing.

\section{Nucleotide Accession Number(s)}

The nucleotide sequence of FONA-7 ESBL, from $S$. fonticola PE1 strain, has been deposited in the GenBank database under accession number MN634199, and the complete genome sequence of $S$. fonticola strain PE1 has been deposited under BioProject accession number PRJNA579367, and GenBank accession number JAAAXN000000000.1.

\section{Disclosure Statement}

No competing financial interests exist.

\section{Funding Information}

This study was supported by Comisión Nacional de Investigación Científica y Tecnológica (Grant No. CONICYT $\mathrm{BCH}$ 72170436) and Conselho Nacional de Desenvolvimento Científico e Tecnológico (CNPq; Grant Nos. 433128/ 2018-6 and 312249/2017-9). A.C.-A. is a research grant fellow of Coordenação de Aperfeiçoamento de Pessoal de Nível Superior (PNPD/CAPES; Grant No. 88887.463414/ 2019-00). N.L. and J.C.C.-D. are research fellows of CNPq (Process No. 312249/2017-9 and 304999-18).

\section{Supplementary Material}

Supplementary Table S1

\section{References}

1. Aljorayid, A., R. Viau, L. Castellino, and R.L.P. Jump. 2016. Serratia fonticola, pathogen or bystander? A case series and review of the literature. IDCases. 5:6-8.

2. Stock, I., S. Burak, K.J. Sherwood, T. Grüger, and B. Wiedemann. 2003. Natural antimicrobial susceptibilities of strains of "unusual" Serratia species: S. ficaria, S. fonticola, S. odorifera, S. plymuthica and S. rubidaea. J. Antimicrob. Chemother. 51:865-885.

3. Naas, T., L. Poirel, and P. Nordmann. 2008. Minor extended-spectrum $\beta$-lactamases. Clin. Microbiol. Infect. 14(Suppl. 1):42-52. 
4. Philippon, A., P. Slama, P. Dény, and R. Labia. 2016. A structure-based classification of class A $\beta$-lactamases, a broadly diverse family of enzymes. Clin. Microbiol. Rev. 29:29-57.

5. Clinical and Laboratory Standards Institute. 2020. Performance Standards for Antimicrobial Susceptibility Testing; Twenty-Nine Informational Supplement. CLSI document M100-S30. CLSI, Wayne, PA.

6. Clinical Laboratory Standards Institute. 2015. Performance Standards for Antimicrobial Disk and Dilution Susceptibility Tests for Bacteria Isolated from Animals. 3rd ed. CLSI Supplement VET01S. CLSI, Wayne, PA.

7. Dunne, W.M., and D.J. Hardin. 2005. Use of several inducer and substrate antibiotic combinations in a disk approximation assay format to screen for AmpC induction in patient isolates of Pseudomonas aeruginosa, Enterobacter spp., Citrobacter spp., and Serratia spp. J. Clin. Microbiol. 43:5945-5949.

8. Bankevich, A., S. Nurk, D. Antipov, A.A. Gurevich, M. Dvorkin, A.S. Kulikov, V.M. Lesin, S.I. Nikolenko, S. Pham, A.D. Prjibelski, A.V. Pyshkin, A.V. Sirotkin, N. Vyahhi, G. Tesler, M.A. Alekseyev, and P.A. Pevzner. 2012. SPAdes: a new genome assembly algorithm and its applications to single-cell sequencing. J. Comput. Biol. 19: 455-477.

9. Zankari, E., H. Hasman, S. Cosentino, M. Vestergaard, S. Rasmussen, O. Lund, F.M. Aarestrup, and M.V. Larsen. 2012. Identification of acquired antimicrobial resistance genes. J. Antimicrob. Chemother. 67:2640-2644.

10. Carattoli, A., E. Zankari, A. García-Fernández, M. Voldby Larsen, O. Lund, L. Villa, F. Møller Aarestrup, and H. Hasman. 2014. In silico detection and typing of plasmids using PlasmidFinder and plasmid multilocus sequence typing. Antimicrob. Agents Chemother. 58:3895-3903.

11. Schwede, T. 2003. SWISS-MODEL: an automated protein homology-modeling server. Nucleic Acids Res. 31:33813385.

12. Krieger, E., T. Darden, S.B. Nabuurs, A. Finkelstein, and G. Vriend. 2004. Making optimal use of empirical energy functions: force-field parameterization in crystal space. Proteins Struct. Funct. Bioinforma. 57:678-683.

13. Bramucci, E., A. Paiardini, F. Bossa, and S. Pascarella. 2012. PyMod: sequence similarity searches, multiple sequence-structure alignments, and homology modeling within PyMOL. BMC Bioinformatics. 13(Suppl. 4):S2.
14. Guenther, S., C. Ewers, and L.H. Wieler. 2011. Extendedspectrum beta-lactamases producing $E$. coli in wildlife, yet another form of environmental pollution? Front. Microbiol. 2:246.

15. Dolejska, M., and I. Literak. 2019. Wildlife is overlooked in the epidemiology of medically important antibioticresistant bacteria. Antimicrob. Agents Chemother. 63:1-5.

16. Liakopoulos, A., B. Olsen, Y. Geurts, K. Artursson, C. Berg, D.J. Mevius, and J. Bonnedahl. 2016. Molecular characterization of extended-spectrum-cephalosporinresistant Enterobacteriaceae from wild kelp gulls in South America. Antimicrob. Agents Chemother. 60:6924-6927.

17. Mohsin, M., S. Raza, K. Schaufler, N. Roschanski, F. Sarwar, T. Semmler, P. Schierack, and S. Guenther. 2017. High prevalence of CTX-M-15-type ESBL-producing E. coli from migratory avian species in Pakistan. Front. Microbiol. 8:2476.

18. Skeel, M.A., and E.P. Mallory. 1996. Whimbrel (Numenius phaeopus), version 2.0. In A.F. Poole and F.B. Gill (eds.), The Birds of North America. Cornell Lab of Ornithology, Ithaca, NY.

19. González-Candelas, F., C. Francés-Cuesta, and N. GarcíaGonzález. 2019. The power and limitations of genomic surveillance of bacteria. Future Microbiol. 14:1345-1348.

20. Su, M., S.W. Satola, and T.D. Read. 2019. Genome-based prediction of bacterial antibiotic resistance. J. Clin. Microbiol. 57:e01405-18.

21. Ellington, M.J., O. Ekelund, F.M. Aarestrup, R. Canton, M. Doumith, C. Giske, H. Grundman, H. Hasman, M. Holden, K.L. Hopkins, J. Iredell, G. Kahlmeter, C.U. Köser, A. MacGowan, D. Mevius, M. Mulvey, T. Naas, T. Peto, J. M. Rolain, Ø. Samuelsen, and N. Woodford. 2017. The role of whole genome sequencing in antimicrobial susceptibility testing of bacteria: report from the EUCAST Subcommittee. Clin. Microbiol. Infect. 23:2-22.

Address correspondence to: Nilton Lincopan, PhD

Department of Microbiology Institute of Biomedical Sciences University of São Paulo São Paulo 05508-900

Brazil

E-mail: lincopan@usp.br 